\title{
STANDARDISING MAINTENANCE JOBS TO IMPROVE GROUPING DECISION MAKING
}

\author{
Agergaard, Julie Krogh; \\ Sigsgaard, Kristoffer Vandrup; \\ Mortensen, Niels Henrik; \\ Ge, Jingrui; \\ Hansen, Kasper Barslund; \\ Khalid, Waqas
}

Technical University of Denmark

\begin{abstract}
Maintenance decision making is an important part of managing the costs, effectiveness and risk of maintenance. One way to improve maintenance efficiency without affecting the risk picture is to group maintenance jobs. Literature includes many examples of algorithms for the grouping of maintenance activities. However, the data is not always available, and with increasing plant complexity comes increasingly complex decision requirements, making it difficult to leave the decision making up to algorithms.

This paper suggests a framework for the standardisation of maintenance data as an aid for maintenance experts to make decisions on maintenance grouping. The standardisation improves the basis for decisions, giving an overview of true variance within the available data. The goal of the framework is to make it simpler to apply tacit knowledge and make right decisions.

Applying the framework in a case study showed that groups can be identified and reconfigured and potential savings easily estimated when maintenance jobs are standardised. The case study enabled an estimated $7 \%-9 \%$ saved on the number of hours spent on the investigated jobs.
\end{abstract}

Keywords: Modularization, Maintenance, Knowledge management, Large-scale engineering systems, Big data

\section{Contact:}

Agergaard, Julie Krogh

Technical University of Denmark

Mechanical Engineering

Denmark

jkrag@mek.dtu.dk

Cite this article: Agergaard, J. K., Sigsgaard, K. V., Mortensen, N. H., Ge, J., Hansen, K. B., Khalid, W. (2021)

'Standardising Maintenance Jobs to Improve Grouping Decision Making', in Proceedings of the International Conference on Engineering Design (ICED21), Gothenburg, Sweden, 16-20 August 2021. DOI:10.1017/pds.2021.531 


\section{INTRODUCTION}

Larger product programs with increasingly complex products are increasing the importance of maintenance efforts. It is important to make the right decisions, as inefficient maintenance introduces avoidable costs, loss in quality, and production downtime. Maintenance efforts also affect the safety of employees and the environment, making it critical to manage the risks involved in maintenance (BSI: British Standards, 2016). The grouping of maintenance actions is an optimization that can improve the amount of time spent on maintenance without changing the core maintenance actions. When the core maintenance actions are not changed the risk picture is not affected, as grouping takes advantage of the overlaps in maintenance. This can be shared support actions or overlapping down time. Grouping methods have been suggested in both manufacturing and maintenance literature. These methods are most commonly focused on algorithms that analyse the maintenance and suggest optimized solutions (Assaf and Shanthikumar, 1987; Cui and Li, 2006; Dekker and Smeitink, 1991; Hu and Zhang, 2014; Li et al., 2018; Navaei and ElMaraghy, 2014; Nzukam et al., 2017; Poppe et al., 2018; Van et al., 2013; Vu et al., 2018, 2014; Wu et al., 2020; Zhang et al., 2017). However, the articles have not focused on creating methods and frameworks for more complex decisions that require human expert evaluation. Martínez et al. (2013) mention how maintenance management generally lacks simple tools that can illustrate patterns of deficiencies in a clear and simple way. Similar conclusions were drawn when attempting to implement grouping methods in a case company. The internal experts in the case company carried tacit knowledge crucial to the grouping of maintenance activities. This made it impossible to perform grouping solely with data, thus making it implausible not to include the experts in the grouping decision making process. This paper therefore draws on experience from product architecture literature to suggest a framework for mapping out available maintenance job data. The goal of the mapping is to support experts with available data in the grouping decision making process, allowing the experts to make a more informed decision. The mapping is focused on achieving a standardised format that shows true similarities and variation to ensure that identified groups are plausible. The parallel to product architecture theory and production planning is drawn to cover the gap in maintenance grouping decision making frameworks. Product architecture literature has several examples of mapping out a product program to achieve an overview of true variation (Harlou, 2006; Løkkegaard et al., 2016; Meyer and Lehnerd, 1997; Voss and Hsuan, 2009). The framework seeks to achieve a similar overview of the maintenance jobs to identify opportunities for grouping.

The paper first introduces the research method utilized, then summarizes relevant literature in the second and third sections. The fourth section introduces the proposed framework, and the fifth section summarizes the use of the framework in a case study. This is followed by a discussion of the method compared to literature and the results from the case study in the sixth and seventh sections.

\section{RESEARCH METHOD}

The study described in this paper is based on a literature study and a case study. The framework suggested in this paper was built using knowledge gained from literature and from knowledge about maintenance in industry from collaboration with a case company. The initial goal of the literature study was to identify methods for grouping in the case company. However, the identified methods were not applicable with the data available in the case company: the data was insufficient for algorithms and too complex and spread out for manual grouping in its as-is format. Methods for mapping out complexly structured information through standardisation are prevalent in the product world - specifically in product architecture development. The literature study was therefore expanded with methods for standardisation from the product architecture development.

The case company operates a multitude of large offshore plants. The case study and the proposed framework were developed based on the data from the company's maintenance management system along with knowledge gained from meetings, workshops and semi-structured interviews with company experts such as engineers, maintenance responsibles, etc. The quantifiable part of the data is a part of a data model with data on the maintenance from 2010 - 2019. The data model was built using data from the company's Computerized Maintenance Management System (CMMS) over a period of a year. The model is continuously maintained and verified in collaboration with the case company. The collaboration has allowed validation of the framework throughout the iterations of development. 


\section{LITERATURE}

This section introduces literature relevant to the grouping of maintenance and how issues of standardisation were solved in the product world. The first section introduces maintenance and its importance to product manufacturing. The second section introduces grouping methods from literature. As the grouping methods were not applicable with the data available from the case company, the final section introduces methods for standardisation from the product world.

\subsection{Maintenance for improved manufacturing}

Maintenance is an important cost factor in manufacturing. It enables continuous production at high quality and without safety risks to people and the environment. The goal of maintenance is to ensure the following objectives (Dansk Standard, 2017): Availability of the item at the required function and optimum costs; The safety of people and the environment; Any impact on the environment is secured; Durability of the item and quality of the product/service provided. A failure of an item is classified as an inability to perform the required function. Maintenance can either be the repair of a failed item or in the effort to prevent future possible failures. When repairing a failed item it is called corrective maintenance $(\mathrm{CM})$. When performing maintenance to prevent future possible failures it is called preventive maintenance (PM) (Dansk Standard, 2017). With increasingly complex production facilities, the maintenance complexity rises. This makes decision making increasingly challenging.

Maintenance data is stored in a Computerized Maintenance Management System (CMMS). A variety of factors affect the quality of the maintenance data collected in the CMMS. The often poorer quality of the maintenance data makes analyses often based on industry databases or broad assumptions. However, it is possible to clean and structure the data already available. Cleaning and utilizing the available data gives insight into actual maintenance practices without the added uncertainty of databases and assumptions (Hodkiewicz and Ho, 2016). Hodkiewicz and Ho (2016) mention how cleaning of historical data for use in maintenance analyses has been overlooked, and introduces a method using a rule-based approach. The proposed approach is shown to be repeatable and can be applied to a large amount of available data.

\subsection{Grouping methods}

Grouping of products by their manufacturing operations has been investigated by numerous authors. The methods base the grouping on a variety of criteria and apply an algorithm that groups the products (Navaei and ElMaraghy, 2014). Similarly, the grouping of maintenance operations is a broad subject and it has been developed and implemented in a variety of engineering systems. The studies take advantage of the fact that maintenance is cheaper to perform together than individually ( $\mathrm{Vu}$ et al., 2014). The lowered costs are due to the dependencies between the equipment (Nzukam et al., 2017). In opportunistic maintenance, a failure is seen as an opportunity to do other work (Nzukam et al., 2017).This makes a failure an opportunity to perform any upcoming preventive maintenance at the location (Cui and Li, 2006; Zhang et al., 2017). However, always performing preventive maintenance at given opportunities sacrifices plannable maintenance (Cui and Li, 2006). Other methods suggest evaluating the condition before deciding whether to do opportunistic maintenance ( $\mathrm{Hu}$ and Zhang, 2014; Poppe et al., 2018) or grouping based on a predetermined replacement threshold and replacing all if one fails (Dekker and Smeitink, 1991; Wu et al., 2020).

Other grouping methods suggest optimizing the maintenance plans for the individual pieces of equipment and then grouping based on shared intervals (Van et al., 2013; Vu et al., 2018). Li et al. (2018) suggest grouping production equipment maintenance by the criticality of the machines. Assaf and Shanthikumar (1987) suggest grouping corrective maintenance jobs until a threshold number of components in the group has failed and then replacing all pieces at once.

Several authors use one or more criteria for grouping. The criteria are the basis for making a decision on whether grouping maintenance jobs is possible. The identified criteria are summarized in Table 1.

As can be seen from the table, few criteria are shared across the studies. 
Table 1. Summary of the mention of criteria in literature.

\begin{tabular}{|l|c|c|c|c|c|c|c|}
\hline Criteria & $\begin{array}{l}\text { (Nzukam } \\
\text { et al., } \\
\text { 2017) }\end{array}$ & $\begin{array}{l}\text { (Dekker } \\
\text { et al., } \\
\text { 1997) }\end{array}$ & $\begin{array}{l}\text { (Hu and } \\
\text { Zhang, } \\
\text { 2014) }\end{array}$ & $\begin{array}{l}\text { (Guo et } \\
\text { al., 2018) }\end{array}$ & $\begin{array}{l}\text { (Van et } \\
\text { al., 2013) }\end{array}$ & $\begin{array}{l}\text { (Assaf and } \\
\text { Shanthiku } \\
\text { mar, 1987) }\end{array}$ & $\begin{array}{l}\text { (Li et } \\
\text { al., } \\
\text { 2018) }\end{array}$ \\
\hline $\begin{array}{l}\text { Structural } \\
\text { dependencies }\end{array}$ & $\mathrm{X}$ & $\mathrm{X}$ & & & & & \\
\hline $\begin{array}{l}\text { Failure probability } \\
\text { distribution }\end{array}$ & $\mathrm{X}$ & & & & & & \\
\hline Position & & & $\mathrm{X}$ & & & & \\
\hline The repair style & & & $\mathrm{X}$ & & & & \\
\hline $\begin{array}{l}\text { Maintenance and } \\
\text { industry standards }\end{array}$ & & & $\mathrm{X}$ & & & & \\
\hline $\begin{array}{l}\text { Economic } \\
\text { dependence }\end{array}$ & & $\mathrm{X}$ & & $\mathrm{X}$ & & & \\
\hline $\begin{array}{l}\text { Stochastic } \\
\text { dependence }\end{array}$ & & $\mathrm{X}$ & & $\mathrm{X}$ & & & \\
\hline Execution date & & & & $\mathrm{X}$ & $\mathrm{X}$ & & \\
\hline Operations & & & & $\mathrm{X}$ & $\mathrm{X}$ & & \\
\hline Maintenance duration & & & & & $\mathrm{X}$ & & \\
\hline $\begin{array}{l}\text { Number of failed } \\
\text { components }\end{array}$ & & & & & & $\mathrm{X}$ & \\
\hline Shared resources & & & & $\mathrm{X}$ & & & \\
\hline Downtime & & & & $\mathrm{X}$ & & & \\
\hline Criticality & & & & & & & $\mathrm{X}$ \\
\hline
\end{tabular}

\subsection{Standardisation in the product world}

In attempting to apply the methods for grouping presented in literature it was discovered that the data quality was not sufficient. Based on a call for more research by Ruschel et al. (2017) and for simpler tools by Martínez et al. (2013), it is clear that others have come to a similar conclusion. However, the raw data format of the maintenance data is also not useful for grouping, as the complexity is too great. Complexity has successfully been managed through modularisation and standardisation in product family management and simple graphical tools for decision making are not new in the product world. By introducing modularisation and standardisation principles, companies manage to handle large, complex product assortments without losing the amount of variation offered. In modularisation, products are constructed from predefined modules with set interfaces. Variants are then introduced within the modules instead of creating a whole new product when introducing a new feature. The key to modularisation is keeping up the standardised modules and interfaces, and ensuring that these do not change. The benefits of modularisation of product families include shorter time-to-market, standardisation and automation of production, and more (Harlou, 2006; Meyer and Lehnerd, 1997). Løkkegaard et al. (2016) present a conceptual model for the modularisation of services. A case study of the conceptual model showed that an engineering company could improve their service delivery process by re-using well-defined standardised modules. Maintenance operations are similar to services in that they are intangible actions compared to the physicality of their product counterparts. The study by Løkkegaard et al. (2016) is therefore an indication that theories and methods of modularisation and standardisation are applicable in maintenance management.

\section{MAPPING OUT STANDARDISED MAINTENANCE JOBS}

The proposed framework aids the mapping and standardisation of maintenance jobs with the goal of grouping maintenance jobs. The goal of the framework is to provide sufficient knowledge in a readable format for situations where the decisions must be made by an expert human. That is, a situation where some of the knowledge required to make the decision is tacit and thus cannot be represented by data in an algorithm or other computerised methods. As shown in the literature review, several methods have been proposed for the clustering of maintenance (Assaf and Shanthikumar, 
1987; Cui and Li, 2006; Dekker and Smeitink, 1991; Hu and Zhang, 2014; Li et al., 2018; Nzukam et al., 2017; Poppe et al., 2018; Van et al., 2013; Vu et al., 2014, 2018; Wu et al., 2020; Zhang et al., 2017). However, these methods tend to not account for imperfect and unavailable data. This is a general trend in maintenance research, as also concluded by Hodkiewicz and Ho (2016). The framework presented in this paper seeks to instead standardise the available maintenance data to deliver an overview of the maintenance that shows true variation, allowing the grouping of the maintenance jobs. Using standardisation as a means to achieve an overview of true variation is based on experiences from product architecture research (Harlou, 2006; Løkkegaard et al., 2016; Meyer and Lehnerd, 1997).

The framework is based on a matrix format where all of the maintenance job data is mapped out. When the maintenance job data is represented in one standardized, cohesive overview it is possible to identify possible overlaps within the maintenance. Such overlaps indicate the possibility of a group, allowing the analysing maintenance expert to more easily determine groups of maintenance jobs amongst the planned maintenance. This section introduces the framework and describes how the information can be mapped out.

In order for an expert to be able to group the jobs, they must be standardised. Without standardisation, similarities might seem like variations only due to variations in the format of the knowledge. The framework is based on a matrix format with jobs as columns and rows with criteria describing the jobs (Figure 1). The criteria represent different types of knowledge that are important to the comparison of maintenance jobs. The jobs are then compared along the reading direction and the contents of the individual jobs can be gauged in the vertical direction.

\begin{tabular}{r|cccc} 
Jobs & 1 & 2 & 3 & $\ldots$ \\
$\begin{array}{r}\text { Criteria Location } \\
\text { (pipe section) }\end{array}$ & $5 \mathrm{~B}$ & $5 \mathrm{~B}$ & $4 \mathrm{~A}$ & \\
Resources & New filter & $\begin{array}{c}\text { New 0- } \\
\text { ring }\end{array}$ & Lube-oil \\
Operations & Replace & Replace & Refill \\
Execution date & $01 / 01$ & $01 / 01$ & $02 / 01$
\end{tabular}

\begin{tabular}{|c|c|c|c|}
\hline Jobs & 1 & 2 & 3 \\
\hline $\begin{array}{l}\text { Criteria } \\
\text { Location } \\
\text { (pipe section) }\end{array}$ & $5 B$ & $5 B$ & $4 \mathrm{~A}$ \\
\hline Resources & New filter & $\begin{array}{l}\text { New O- } \\
\text { ring }\end{array}$ & Lube-oil \\
\hline Operations & Replace & Replace & Refill \\
\hline Execution date & 01/01 & 01/01 & $02 / 01$ \\
\hline
\end{tabular}

\section{Figure 1. The matrix format is the basis of the framework. The criteria used are inspired by those presented in literature (section 3.2). LEFT: Each order is mapped out, showing true variations and similarities. RIGHT: The green box indicates an identified group.}

An important factor of the matrix format is the standardisation of the job criteria. The criteria are the factors that describe the job. In Figure 1, each of the example criteria have been mapped out and standardised for each of the orders. The criteria then indicate similarities in the data. In the example matrix on the right a possible group of jobs 1 and 2 has been identified. The jobs are performed on the same section of the pipeline on the same and both require the replacement of a component, making them compatible for a grouping. As shown in Table 1, several criteria for grouping have been suggested by a series of authors. This indicates that not one set of criteria can be used to identify maintenance groups in all cases. Based on experiences from the study, the steps shown in Figure 2 are suggested for the development of the overview shown in Figure 1. The following section instead describes how the criteria can be identified and the information can mapped out.

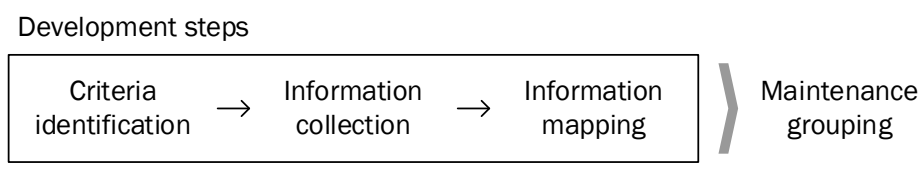

Figure 2. Suggested steps for the development of the framework overview.

\subsection{Deciding on and standardising criteria}

As seen from literature, criteria can be operations that describe the actions to be taken along with practical information about the job such as the type of job, the location, the required materials and so forth (Assaf and Shanthikumar, 1987; Dekker et al., 1997; Guo et al., 2018; Hu and Zhang, 2014; Li et al., 2018; Nzukam et al., 2017; Van et al., 2013). Before starting the process of standardisation it is 
important to understand what knowledge is required to group the maintenance jobs in the specific context. A way to achieve such an overview is by creating an initial list of the criteria that can affect the decisions made on clustering. This can be based on the knowledge of internal experts and from research such as the criteria identified in literature summarized in Table 1.

An initial collection of criteria should focus on getting as much on the table as possible, to ensure that the solution space is opened up completely. From the list of criteria, identifying the criteria where historical and/or real time data can be applied can help narrow down the list to obtainable criteria. Historical data can be used to evaluate current groups if relevant, while real-time data is essential to a dynamic, ongoing grouping process. Evaluation of previous clusters can give insight into the possibilities for grouping that reflect the actualized process (Hodkiewicz and Ho, 2016).

The information on the identified criteria must be standardised. Criteria such as order type, skills, resources etc. must be described using shared vocabulary. As an example, it can be ensuring a required skill such as mechanical is always mentioned in the same way. Variations may be MECH, mechanic etc. Standardising operations is likely a longer process, as these are often formulated using a free text format, introducing many variants for the same actions. An action can often be described in many ways and the free text format also introduces variations in abbreviations and typos. An example is the action lubricate, that can be written as Lubricate, Lubrication, Grease, Lubric., Lbricate or more. An important step is therefore to standardise the content of the job to make it possible to compare the actions and effects across all jobs. So for the example of all of the variants of lubrication, the actions are instead described using a standard word, e.g. Lubricate.

Experience with the case company showed that it was necessary to classify operations by the following categories: Support work, Main work, and Document and test. These categories were based on experiences from meetings, semi-structured interviews and workshops with company maintenance experts. The categories help discern actions that influence the equipment from actions that are required to perform the job and to manage the quality of the maintenance. Support work consist of actions such as material collection, scaffolding, isolation and so forth. It is the work required to be able to perform the job and ensure the safety of the employees and not actions that directly influence the equipment. Main work is the maintenance actions that effect the equipment, such as lubrication or replacements. Main work can also be non-life-prolonging, such as visual inspections or function checks. Document and test concerns the work required to keep track of the maintenance and ensure the quality of the performed actions. Documentation is needed to update master data and to keep track of the state of the equipment. Tests are needed to ensure the maintenance was performed correctly and that it had the desired effect.

With the job knowledge standardised and mapped out it is possible to discern similarities across jobs. This makes it possible to identify jobs that share dependencies and grouping them. The standardised mapping gives an overview of the available data so that the maintenance expert must only apply the information that is not available in the CMMS. The formation of the groups can be one of the strategies introduced in the literature section or other grouping strategies that better suit the context.

\section{CASE STUDY}

The framework was implemented and further developed in a grouping project in a case company. The company operates large offshore production facilities where maintenance is one of the largest operational cost factors. Employee and environmental safety and minimization of production loss and maintenance costs are important factors in keeping the business going. These considerations make grouping an interesting area to investigate. The study performed in this paper is a part of a research project that has previously developed a maintenance data model in a Business Intelligence tool. The data model was developed in collaboration with the company over a year. The model includes information on all maintenance jobs registered in the CMMS from 2011-2019. This made it easy to identify and extract the necessary data.

The criteria with available historical data were analysed to get a better understanding of how the criteria describe groups created in the past. The criteria were evaluated by identifying the number of jobs that had previously included two or more values within each group. Each criteria were visualised in a graph similar to the one shown in Figure 3. 


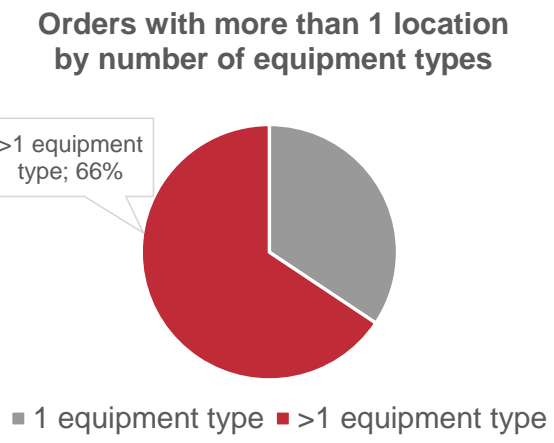

Figure 3. \% of grouped orders with more than 1 equipment type per order. The $66 \%$ indicates that groups can be created across different equipment types.

The resulting visuals were organized on a poster to discuss in a semi-structured workshop with company maintenance experts. The meeting gave insight into why some criteria had groups and some did not. The final list of criteria ended up consisting of criteria where more than $20 \%$ of orders in the scope had been grouped. The analysis of the historical groups yielded the following list of criteria:

- Location: at a minimum, the same platform and system. Criteria requires expert knowledge: closeness of equipment not included in data.

- $\quad$ Equipment type: preferably some shared equipment, but different types can be grouped

- $\quad$ Required skills: preferably compatible skills

- Shutdown requirements: the same or compatible shutdown requirements.

- $\quad$ Support work: preferably shared support work to minimize number of isolations, scaffolds etc.

- Main work: for some main work, repeating the same work several times in a row can improve the time spent.

- Document and test: little influence of document and test actions expected, as time spent on these steps is already small. However, information included to ensure full picture.

- Order dates: overlapping or negligible time windows required. No critical time windows moved for the sake of grouping.

The operations were mapped out into action words as described in section 4.1. The 36 orders and 98 distinct operation descriptions were standardized into 54 action words, simplifying the action description. With the full knowledge about the grouping criteria, the maintenance jobs were mapped out in a matrix format similar to the concept matrix in Figure 1.

\subsection{The resulting standardized maintenance jobs}

The criteria were all mapped out in a matrix format on a poster with all of the jobs as columns and the criteria as the rows. An example of how the maintenance jobs were mapped out is shown in Figure 4. As the scope only included orders on the same plant and system, the location criteria was not included in the matrix. Each of the remaining criteria were inserted as rows. Equipment and order dates were visualized with icons for easier comparability. As this analysis was performed on historical jobs, the actual time posting days were also included. This indicates the day on which the job was actually performed. The other dates used for the mapping includes the start and finish of the allowed window for the job, indicating how much the job could have been moved to fit a group. The shutdown requirement criteria was simplified to yes or nothing, as all shutdowns required for the jobs in the scope were for the full system. The action words were split into support, main, and document and test. All actions taken in the scope are listed on the left hand side to improve identification of possible action overlaps. For example, the analyser can find the row describing isolations from the list and quickly identify orders with that action by moving vertically along the word axis.

The matrix format of the mapping enabled faster comparisons of orders, as any similarities in the data were easily spotted. With the standardised content, the groups were easily formulated and the saved actions were clear from the duplicates in the data. 


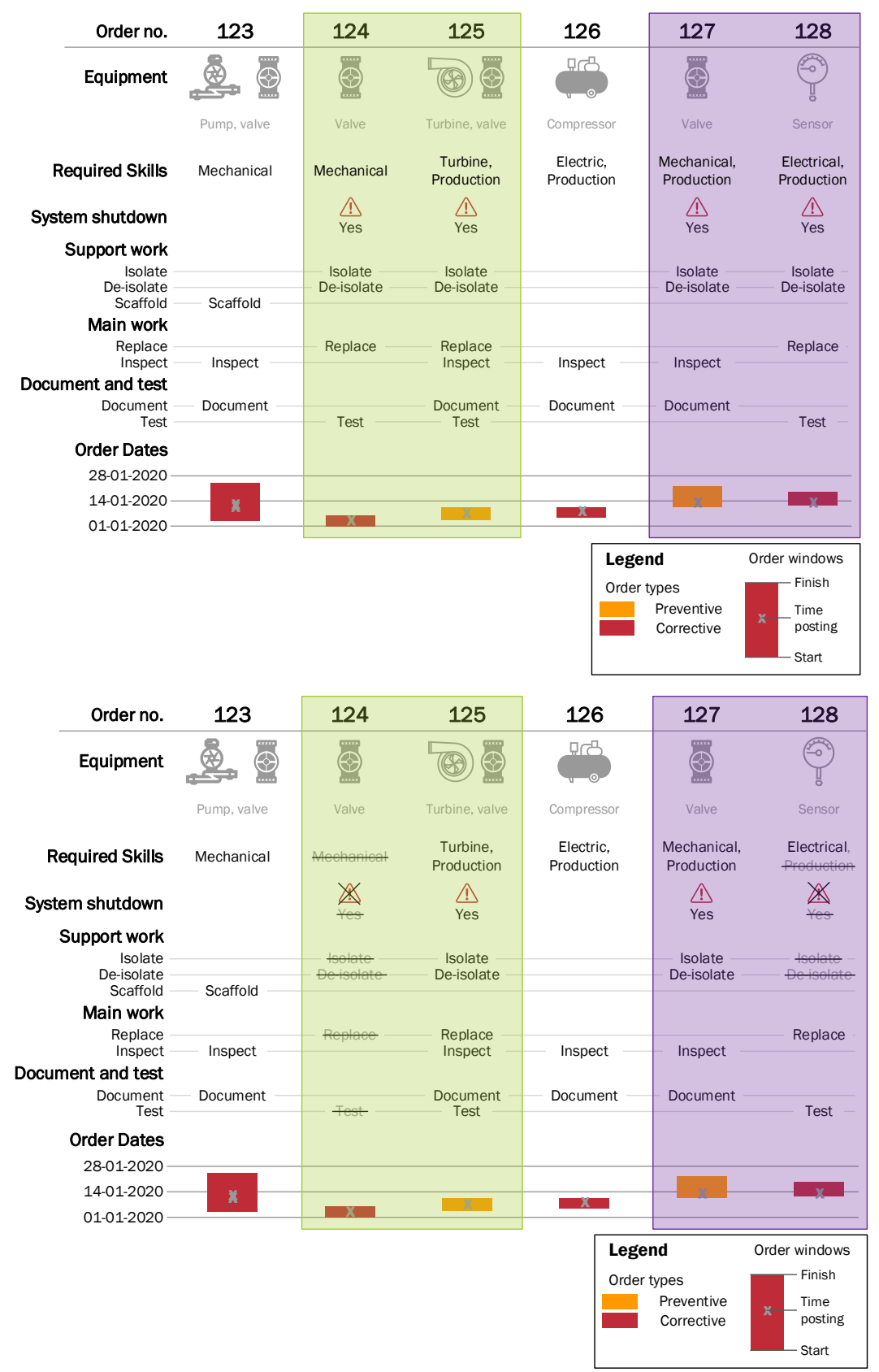

Figure 4. An example of how the final poster looked. TOP: The green (jobs $124+125$ ) and purple (jobs $127+128$ ) boxes indicate the identified groups across the example jobs.

BOTTOM: The saved actions and shutdowns.

As shown in Figure 4, Bottom, the combination of jobs $124+125$ and $127+128$ means saving two system shutdowns, two times isolation and de-isolation, a replacement and a test. As the valve is already being preventively replaced in job 125, the actions in job 124 are unnecessary. The mechanical skill is not required after the grouping, as it was clear from job 125 that the turbine workers could take care of the work required on the valves. Similarly, the production skills are only required once for the purple group, as the isolation and de-isolation from job 128 are saved.

For the full scope, the mapping allowed an identification of four possible groups out of the 36 orders in the full scope within a short period of time. This type of identification had not been available had the orders not been mapped out using the method. Prior to the mapping, the information was purely available in separate tables and the operations were not comparable on a scale larger than 2-3 orders at a time. Any groups that might occur with the current process happen by chance. This type of optimization would only occur in the case company if two compatible jobs happened to be placed close enough that a maintenance employee noticed it when it was time to execute the jobs. With the 
framework implemented within the maintenance planning process it would be possible to identify the groups in a structured way.

Based on the mapped out job actions, the time saved from the groups was estimated at $7 \%$ when grouping within job types and $9 \%$ when grouping across order types. The estimate is based on the average amount of time spent historically on the no longer needed actions. For repeated actions it was assumed that the amount of time the action was planned to take was improved by $10 \%$ every time the action was repeated. As an example, the calculation for the green group in Figure 4, Bottom would be:

$$
\begin{aligned}
\text { Estimated time }[h] & \\
& =\text { Current jobs } 124+125[h]-\text { avg isolate }[h]-\text { avg deisolate }[h] \\
& - \text { avg replace }[h]-\text { avg test }[h]
\end{aligned}
$$

In total, 42 work hours were estimated saved within the study scope when grouping within maintenance types and 57 work hours were estimated saved when grouping across maintenance types. The number of orders was improved $11 \%$ within order types and 19\% across order types. A decrease in the number of orders is expected to improve the number of orders that must be fit in a schedule as well as the number of times the production is shutdown, thus saving on ramp up and down time of the production equipment.

\section{DISCUSSION}

The presented framework helped group jobs which improved the amount of planned hours by 7\%-9\% for a smaller scope at a case company. The framework supports the standardisation and mapping of maintenance jobs in a way that allows an expert engineer to evaluate the available information about the jobs and apply their own tacit knowledge to take decisions on grouping that were not previously possible. This could be situations where the information required to run an algorithm is not available and the decision must be made by an expert or when the data quality is not sufficient to achieve a useful result. In the case company, the available data did not cover all aspects required to run an algorithm and nor was the data available in a collective overview. This meant that maintenance experts were not able to make informed decisions about the grouping of maintenance. The standardized criteria lend themselves to a simple, graphical format that allows quicker identification of patterns of similarities or variations. As such it seeks to fulfil part of the gap expressed by Martínez et al. (2013). As concluded in this study and as mentioned by Hodkiewicz and Ho (2016), there is a lot of untapped potential in the currently available maintenance data. By mining and cleaning the historical data it is possible to gain insights that were not available at the current format. The cleaning process similarly typically takes less time than collecting new data and as shown by Hodkiewicz and Ho (2016) can be repeated for a larger scope if necessary. The future of grouping will be simplified when the collected data is suitable and tacit knowledge is mapped out. However, data collection is typically a lengthy process and it is difficult to achieve an overview that suits all aspects of maintenance analysis performed in a production company (Hodkiewicz and Ho, 2016), likely leaving a need for some sort of data mapping or cleaning in maintenance grouping.

The study indicates the applicability of standardisation principles from modularisation theory on the handling of maintenance variation and complexity. As such it calls for more investigation of the applicability of design and modularisation methods in maintenance management research. Future studies should focus on the scalability of the method to all of the maintenance data at a case company. More studies on the applicability of the framework in daily work would also allow insight into the usefulness of the framework within a dynamic grouping environment.

\section{CONCLUSION}

The framework developed in the research study presented in this paper enables the grouping of maintenance activities. Grouping is an optimization effort that can improve the amount of time spent on maintenance without changing the risk picture of the maintenance. Methods suggested in literature requires a data quality that is not always available in companies, as information is often tacit among the internal maintenance experts. This makes it necessary to have an expert making the decision, making it difficult to create an algorithm that solves the issue as suggested in most of the identified literature. To better present the available data to enable these expert to take better decisions, the 
maintenance job grouping criteria were proposed standardised and mapped out. As seen from the case study, such a mapping enabled the identification of similarities across a scope of maintenance jobs in a case study. In the case study, a 7\%-9\% improvement of the planned hours was estimated.

\section{REFERENCES}

Assaf, D. and Shanthikumar, G.J. (1987), “Optimal group maintenance policies with continuous and periodic inspections", Management Science, Vol. 33 No. 1, pp. 1440-1452.

BSI: British Standards. (2016), "ISO 14224:2016".

Cui, L. and Li, H. (2006), "Opportunistic maintenance for multi-component shock models", Mathematical Methods of Operations Research, Vol. 63 No. 3, pp. 493-511.

Dansk Standard. (2017), DS/EN 13306:2017 Maintenance - Maintenance Terminology.

Dekker, R. and Smeitink, E. (1991), “Opportunity-based block replacement”, European Journal of Operational Research, Vol. 53 No. 1, pp. 46-63.

Dekker, R., Wildeman, R.E. and Van Der Duyn Schouten, F.A. (1997), “A review of multi-component maintenance models with economic dependence", Mathematical Methods of Operations Research, PhysicaVerlag, Vol. 45 No. 3, pp. 411-435.

Guo, C., Lyu, C., Chen, J. and Zhou, D. (2018), “A multi-event combination maintenance model based on event correlation”, PLoS ONE, Public Library of Science, Vol. 13 No. 11, available at:https://doi.org/10.1371/journal.pone.0207390.

Harlou, U. (2006), Developing Product Families Based on Architectures, Contribution to a Theory of Product Families.

Hodkiewicz, M. and Ho, M.T.W. (2016), "Cleaning historical maintenance work order data for reliability analysis”, Journal of Quality in Maintenance Engineering, Emerald Group Publishing Ltd., Vol. 22 No. 2 , pp. 146-163.

$\mathrm{Hu}$, J. and Zhang, L. (2014), "Risk based opportunistic maintenance model for complex mechanical systems", Expert Systems with Applications, Vol. 41 No. 6, pp. 3105-3115.

Li, G., Li, Y., Zhang, X., Hou, C., He, J., Xu, B. and Chen, J. (2018), "Development of a preventive maintenance strategy for an automatic production line based on group maintenance method", Applied Sciences (Switzerland), MDPI AG, Vol. 8 No. 10, available at:https://doi.org/10.3390/app8101781.

Løkkegaard, M., Mortensen, N.H. and McAloone, T.C. (2016), “Towards a framework for modular service design synthesis”, Research in Engineering Design, Springer London, Vol. 27 No. 3, pp. 1-13.

Martínez, L.B., Márquez, A.C., Gunckel, P.V. and Andreani, A.A. (2013), "The graphical analysis for maintenance management method: A quantitative graphical analysis to support maintenance management decision making", Quality and Reliability Engineering International, Vol. 29 No. 1, pp. 77-87.

Meyer, M.H. and Lehnerd, A.P. (1997), The Power of Product Platforms, The Free Press.

Navaei, J. and ElMaraghy, H. (2014), "Grouping product variants based on alternate machines for each operation”, Procedia CIRP, Vol. 17, Elsevier B.V., pp. 61-66.

Nzukam, C., Voisin, A., Levrat, E., Sauter, D. and Iung, B. (2017), “A dynamic maintenance decision approach based on maintenance action grouping for HVAC maintenance costs savings in Non-residential buildings", IFAC-PapersOnLine, Elsevier B.V., Vol. 50 No. 1, pp. 13722-13727.

Poppe, J., Boute, R.N. and Lambrecht, M.R. (2018), “A hybrid condition-based maintenance policy for continuously monitored components with two degradation thresholds", European Journal of Operational Research, Elsevier B.V., Vol. 268 No. 2, pp. 515-532.

Ruschel, E., Santos, E.A.P. and Loures, E. de F.R. (2017), "Industrial maintenance decision-making: A systematic literature review", Journal of Manufacturing Systems, Elsevier B.V., Vol. 45, pp. 180-194.

Van, P. Do, Barros, A., Bérenguer, C., Bouvard, K. and Brissaud, F. (2013), "Dynamic grouping maintenance with time limited opportunities", Reliability Engineering and System Safety, Elsevier Ltd, Vol. 120, pp. 51-59.

Voss, C.A. and Hsuan, J. (2009), "Service Architecture and Modularity", Decision Sciences, Vol. 40 No. 3 , pp. 541-569.

Vu, H.C., Do, P. and Barros, A. (2018), “A Study on the Impacts of Maintenance Duration on Dynamic Grouping Modeling and Optimization of Multicomponent Systems", IEEE Transactions on Reliability, Institute of Electrical and Electronics Engineers Inc., Vol. 67 No. 3, pp. 1377-1392.

Vu, H.C., Do, P., Barros, A. and Bérenguer, C. (2014), "Maintenance grouping strategy for multi-component systems with dynamic contexts", Reliability Engineering and System Safety, Vol. 132, pp. 233-249.

Wu, T., Yang, L., Ma, X., Zhang, Z. and Zhao, Y. (2020), "Dynamic maintenance strategy with iteratively updated group information”, Reliability Engineering and System Safety, Elsevier Ltd, Vol. 197, available at:https://doi.org/10.1016/j.ress.2020.106820.

Zhang, C., Gao, W., Guo, S., Li, Y. and Yang, T. (2017), “Opportunistic maintenance for wind turbines considering imperfect, reliability-based maintenance”, Renewable Energy, Elsevier Ltd, Vol. 103, pp. 606-612. 